\title{
Higher-Order Multifractal Detrended Partial Cross-Correlation Analysis for the Correlation Estimator
}

\author{
Keqiang Dong (iD and Xiaojie Gao \\ College of Science, Civil Aviation University of China, Tianjin 300300, China \\ Correspondence should be addressed to Keqiang Dong; hongzhangdong@163.com
}

Received 28 January 2020; Revised 27 April 2020; Accepted 18 May 2020; Published 4 June 2020

Academic Editor: Ning Cai

Copyright (c) 2020 Keqiang Dong and Xiaojie Gao. This is an open access article distributed under the Creative Commons Attribution License, which permits unrestricted use, distribution, and reproduction in any medium, provided the original work is properly cited.

In this paper, we develop a new method to measure the nonlinear interactions between nonstationary time series based on the detrended cross-correlation coefficient analysis. We describe how a nonlinear interaction may be obtained by eliminating the influence of other variables on two simultaneous time series. By applying two artificially generated signals, we show that the new method is working reliably for determining the cross-correlation behavior of two signals. We also illustrate the application of this method in finance and aeroengine systems. These analyses suggest that the proposed measure, derived from the detrended crosscorrelation coefficient analysis, may be used to remove the influence of other variables on the cross-correlation between two simultaneous time series.

\section{Introduction}

There are numerous real-world systems where the output signals are nonstationary and exhibit complex self-correlation or cross-correlation over a broad range of time scales. The output signals can be characterized by power-law correlations. One method, which has proved to be quite useful to detect the degree of interrelation between two stationary variables, is Pearson's correlation coefficient [1]:

$$
r=\frac{\langle(X-\langle X\rangle) \cdot(Y-\langle Y\rangle)\rangle}{\sigma_{X} \cdot \sigma_{Y}}
$$

where $\langle X\rangle$ is the arithmetic average of $X$ and $\sigma_{X}$ is its standard deviation and likewise for $Y$. Proposition of Pearson's correlation coefficient (PCC) has achieved great success in multivariate analysis, such as the principal component analysis [2], random matrix theory [3], and singular value decomposition [4].

Nevertheless, in real-world systems, nonlinear and nonstationary characteristics are present. Therefore, PCC may not be suitable to describe the interrelation between two variables that are nonlinear and nonstationary. For dealing with the drawbacks of PCC, the detrended cross-correlation analysis (DCCA) method and the DCCA coefficient are proposed by Stanley and Podobnik [5, 6]. The advantage of the DCCA method is that it allows the detection of crosscorrelations between noisy signals with embedded polynomial trends, which can mask the true cross-correlations in the fluctuations of signals. The DCCA method is widely applied to measure the cross-correlations in different fields, such as social sciences [7], biology [8], climatology [9], geophysics $[10,11]$, transportation $[12,13]$, seismic signals $[11,14]$, economics [15-20], and aeroengine dynamics [21-24].

Recently, multifractal analysis is one of the major interests for researchers from interdisciplinary domains to uncover the scaling properties and understand the hidden information. Among these researchers, many of them applied the multifractal analysis to meteorology [25-27], electroencephalography [28], and economics [29-31]. Later, as some researchers thought of extending the research of multifractal analysis to the detrended cross-correlations between time series, the multifractal detrended cross-correlation analysis (MFDXA) was proposed [32-34].

The cross-correlation between two variables may be influenced by other variables. Hence, we have to be alert to 
the possibilities of spurious correlation while investigating the cross-correlation. Then, the methods of partial correlation and partial correlation coefficient are therefore proposed to measure the degree of association between two random variables $[35,36]$. The linear effect may be removed using the partial correlation coefficient (partial CC):

$$
r_{X Y, \xi}=\frac{\left\langle\left(X^{\prime}-\left\langle X^{\prime}\right\rangle\right) \cdot\left(Y^{\prime}-\left\langle Y^{\prime}\right\rangle\right)\right\rangle}{\sigma_{X^{\prime}} \cdot \sigma_{Y^{\prime}}}=\frac{r_{X Y}-r_{X \xi} \cdot r_{Y \xi}}{\sqrt{\left(1-r_{X \xi}^{2}\right) \cdot\left(1-r_{Y \xi}^{2}\right)}},
$$

where $X^{\prime}=X-L_{X}(\xi)$ and $L_{X}(\xi)=c_{0}+c_{1} \xi$ to minimize the mean $E\left(X-L_{X}(\xi)\right)^{2}$ and likewise for $Y^{\prime}$. If $n$ additional variables are to be accounted for, say $\xi_{1}, \xi_{2}, \ldots, \xi_{n}$, the $n$ thorder partial CC can be computed by [36]

$$
r_{X Y .12 \cdots n}=\frac{r_{X Y .12 \cdots n-1}-r_{X n .12 \cdots n-1} r_{Y n .12 \cdots n-1}}{\sqrt{\left(1-r_{X n .12 \cdots n-1}^{2}\right)\left(1-r_{Y n .12 \cdots n-1}^{2}\right)}} .
$$

Lately, the detrended partial cross-correlation analysis and multifractal detrended partial cross-correlation analysis (MFDPXA) which can measure cross-correlations between nonlinear time series influenced by common external forces is proposed [37, 38].

In order to remove the spurious correlation and improve the estimation performance for quantifying the intrinsic interactions between two nonstationary time series, this paper proposes the method of $n$ th-order multifractal detrended partial cross-correlation analysis by incorporating the partial correlation coefficient with the multifractal detrended cross-correlation analysis.

The rest of the paper is organized as follows. In the next section, we introduce the multifractal DCCA coefficient method and propose the method of $n$ th-order multifractal detrended partial cross-correlation analysis. In Section 3, we show the data results for the randomly generated dataset and stock and engine dataset by the proposed methods. Finally, we draw some conclusions in Section 4.

\section{Methodologies}

2.1. Multifractal Detrended Partial Cross-Correlation Analysis. For the sake of clarity, we begin with a summary of the multifractal DCCA coefficient algorithm. For two series $\left\{r_{i}(t)\right\}$ and $\left\{r_{j}(t)\right\}$ with equal length $N$, where $t=1,2, \ldots$, $N$, the computational procedure of the multifractal DCCA coefficient is as follows:

Step 1: construct the profile of each series by eliminating the mean value:

$$
\begin{array}{r}
R_{i}(t)=\sum_{k=1}^{t}\left(r_{i}(k)-\left\langle r_{i}\right\rangle\right), \\
R_{j}(t)=\sum_{k=1}^{t}\left(r_{j}(k)-\left\langle r_{j}\right\rangle\right), \\
\quad t=1,2, \ldots, N,
\end{array}
$$

where $\left\langle r_{i}\right\rangle$ and $\left\langle r_{j}\right\rangle$ are the average values of $\left\{r_{i}(t)\right\}$ and $\left\{r_{j}(t)\right\}$, respectively.

Step 2: divide the profiles $\left\{R_{i}(k)\right\}$ and $\left\{R_{j}(k)\right\}$ into $\left.N_{s}=\operatorname{int}(N / s)\right)$ nonoverlapping units of equal length $s$. Considering that $N$ is usually not a multiple of the time scale $s$, we repeat the same procedure by starting from the opposite end of the sequence in order to take the whole series into account. Thus, we obtain $2 N_{s}$ segments of equal length $s$. In this paper, we follow the previous literature practice and set $10 \leq s \leq N / 4$.

Step 3: for each segment $v\left(v=1,2, \ldots, N_{s}\right.$, $\left.N_{s}+1, \ldots, 2 N_{s}\right)$, the local trends $\left\{\widetilde{R}_{i}^{v}(k)\right\}$ and $\left\{\widetilde{R}_{j}^{v}(k)\right\}$ are estimated on the basis of a least-squares fit of the sequences $\left\{R_{i}(k)\right\}$ and $\left\{R_{j}(k)\right\}$, respectively. The corresponding detrended covariance for $v=1,2, \ldots, N_{s}$ is

$$
f_{\mathrm{DCCA}}^{2}(s, v)=\frac{1}{s} \sum_{t=1}^{s}\left(R_{i}^{(v-1) s+t}(t)-\widetilde{R}_{i}^{v}(t)\right)\left(R_{j}^{(v-1) s+t}(t)-\widetilde{R}_{j}^{v}(t)\right),
$$

and for $v=N_{s}+1, N_{s}+2, \ldots, 2 N_{s}$ is

$$
\begin{aligned}
f_{\mathrm{DCCA}}^{2}(s, v)= & \frac{1}{s} \sum_{t=1}^{s}\left(R_{i}^{N-\left(v-N_{s}\right) s+t}(t)-\widetilde{R}_{i}^{v}(t)\right) \\
& \cdot\left(R_{j}^{N-\left(v-N_{s}\right) s+t}(t)-\widetilde{R}_{j}^{v}(t)\right),
\end{aligned}
$$

where $\left\{\widetilde{R}_{i}^{v}(k)\right\}$ and $\left\{\widetilde{R}_{j}^{v}(k)\right\}$ are the fitting polynomials in the segment $v$.

Step 4: calculate the average of multifractal detrended covariance fluctuation function $F_{\text {DCCA }}^{q}(s, v)$ over all segments:

$$
F_{\mathrm{DCCA}}^{q}(s, v)=\left\{\frac{1}{2 N_{s}} \sum_{v=1}^{2 N_{s}}\left[f_{\mathrm{DCCA}}^{2}(s, v)\right]^{q / 2}\right\}^{1 / q} .
$$

Generally, $q$ can take any real value, except zero. For $q=0$, the equation becomes

$$
F_{0}(s)=\exp \left(\frac{1}{2 N_{s}} \sum_{m=1}^{N_{s}} \ln F(s, m)\right)
$$

For $q=2, F_{\mathrm{DCCA}}^{q}(s, v)$ is equal to the detrended crosscorrelation fluctuation function $F_{\text {DCCA }}^{2}(s)$.

Step 5: estimate the multifractal DCCA coefficient:

$$
\rho_{i j}^{q}(s)=\frac{F_{\mathrm{DCCA}}^{q}(s)}{F_{\mathrm{DFA}\left\{r_{i}(t)\right\}}^{q}(s) F_{\operatorname{DFA}\left\{r_{j}(t)\right\}}^{q}(s)} .
$$


For $q=2$, the standard DCCA coefficient $\rho_{i j}(s)$ is retrieved.

Step 6: compute the multifractal detrended partial cross-correlation coefficient between $X$ and $Y$ by eliminating the influence of the controlling variable $\xi_{1}$ on $X$ and $Y$ analogous to the generalization of the correlation coefficient to partial correlation coefficient:

$$
\rho_{X Y, 1}^{q}=\frac{\rho_{X Y}^{q}-\rho_{X 1}^{q} \rho_{Y 1}^{q}}{\sqrt{\left(1-\left(\rho_{X 1}^{q}\right)^{2}\right)\left(1-\left(\rho_{Y 1}^{q}\right)^{2}\right)}}
$$

named the first-order multifractal detrended partial cross-correlation coefficient (first-order MFDPCC coefficient), where $X, Y$ are random variables, $\xi_{1}$ is the controlling variable, and $\rho_{X Y}^{q}, \rho_{X 1}^{q}, \rho_{Y 1}^{q}$ represent the mean of MFDCCA coefficients for $X$ and $Y, X$ and $\xi_{1}$, and $Y$ and $\xi_{1}$, respectively.

For $q=2$, the first-order detrended partial cross-correlation coefficient (first-order DPCC coefficient) is retrieved.

2.2. The nth-Order Multifractal Detrended Partial CrossCorrelation Analysis and n-Controlling-Variables Detrended Partial Cross-Correlation Coefficient. Considering the crosscorrelation between $X$ and $Y$ affected by more than one variable in complex systems, we define the second-order multifractal detrended partial cross-correlation coefficient (second-order MFDPCC coefficient) by using the partial correlation method [36]:

$$
\rho_{X Y, 12}^{q}=\frac{\rho_{X Y, 1}^{q}-\rho_{X 2,1}^{q} \rho_{Y 2,1}^{q}}{\sqrt{\left(1-\left(\rho_{X 1}^{q}\right)^{2}\right)\left(1-\left(\rho_{Y 2,1}^{q}\right)^{2}\right)}},
$$

where $X, Y$ are random variables, controlling variables $\xi_{1}, \xi_{2}$ are not related to each other, and $\rho_{X Y .1}, \rho_{X 2.1}$, and $\rho_{Y 2.1}$ are first-order MFDPCC coefficients.

Generally, the $n$ th-order multifractal detrended partial cross-correlation coefficient ( $n$ th-order MFDPCC coefficient) is as follows:

$$
\rho_{X Y, 12 \cdots n}^{q}=\frac{\rho_{X Y, 12 \cdots n-1}^{q}-\rho_{X n, 12 \cdots n-1}^{q} \rho_{Y n, 12 \cdots n-1}^{q}}{\sqrt{\left(1-\left(\rho_{X n, 12 \cdots n-1}^{q}\right)^{2}\right)\left(1-\left(\rho_{Y n, 12 \cdots n-1}^{q}\right)^{2}\right)}},
$$

where $\rho_{X Y .12 \cdots n-1}^{q}, \rho_{X n .12 \cdots n-1}^{q}$, and $\rho_{Y n .12 \cdots n-1}^{q}$ are $(n-1)$ thorder MFDPCC coefficients and controlling variables $\xi_{1}, \xi_{2}, \ldots, \xi_{n}$ are not related to each other. For $q=2$, the $n$ thorder detrended partial cross-correlation coefficient ( $n$ thorder DPCC coefficient) is retrieved.

In general, the $n$ th-order partial cross-correlation is necessary when these controlling variables $\xi_{1}, \xi_{2}, \ldots, \xi_{n}$ are not related to each other. Nevertheless, in real-world systems, the variables $\xi_{1}, \xi_{2}, \ldots, \xi_{n}$ generated by large number of interacting units are cross-correlated. Therefore, we define the $n$-controlling-variables multifractal detrended partial cross-correlation coefficient ( $n$-variables MFDPCC) by equation (12) for related controlling variables $\xi_{1}, \xi_{2}, \ldots, \xi_{n}$. Note that when the controlling variables $\xi_{1}, \xi_{2}, \ldots, \xi_{n}$ are not related to each other, the $n$-variables MFDPCC is equivalent to the $n$ th-order MFDPCC.

\section{Data and Analysis}

3.1. Two-Component ARFIMA Process. In order to test the robustness of the proposed $n$-controlling-variables MFDPCC coefficient method, power-law cross-correlated time series $\left\{u_{i}\right\}$ and $\left\{v_{i}\right\}$ are generated by using the twocomponent ARFIMA stochastic process in this section $[18,39,40]$. In this model, the series is defined by

$$
\left\{\begin{array}{l}
u_{i}=\left[W U_{i}+(1-W) V_{i}\right]+\varepsilon_{i} \\
v_{i}=\left[W V_{i}+(1-W) U_{i}\right]+\widetilde{\varepsilon}_{i}, \\
U_{i}=\sum_{j=1}^{\infty} a_{j}\left(\rho_{1}\right) u_{i-j}, \\
V_{i}=\sum_{j=1}^{\infty} a_{j}\left(\rho_{2}\right) v_{i-j}
\end{array}\right.
$$

where $\quad a_{j}(\rho)=\Gamma(j-\rho) / \Gamma(-\rho) \Gamma(i+j) \quad(0<\rho<0.5) \quad$ is weight, $W$ is a free parameter to control the coupling strength between $\left\{u_{i}\right\}$ and $\left\{v_{i}\right\}(0.5 \leq W \leq 1)$, and $\varepsilon_{i}$ and $\widetilde{\varepsilon}_{i}$ are independent and identically distributed (i.i.d.) Gaussian variables with $\left\langle\varepsilon_{i}\right\rangle=\left\langle\widetilde{\varepsilon}_{i}\right\rangle=0$ and $\left\langle\varepsilon_{i}{ }^{2}\right\rangle=\left\langle\widetilde{\varepsilon}_{i}{ }^{2}\right\rangle=$ $1[18,39]$. For different values of $W$, the different coupling strength between the variables $\left\{u_{i}\right\}$ and $\left\{v_{i}\right\}$ is $1-W$. In this section, the two-component ARFIMA series $\left\{u_{i}\right\}$ and $\left\{v_{i}\right\}$ with parameter $\rho_{1}=\rho_{2}=0.3$ and $W=0.5$, denoted by $X$ and $Y$, are employed to detect the interactions between two time series. Then, the effect of white noise sequence $\xi_{1}$ on the cross-correlation of the two series $X$ and $Y$ is tested to investigate the validity of the $n$-controlling-variables MFDPCC coefficient analysis mentioned in this paper. For this purpose, we study the difference between the mean of the MFDCCA coefficient and the $n$-controlling-variables MFDPCC coefficient for any parameter $q$ by using the influence degree function $I(n, q)$. The influence degree function is defined as

$$
I(n, q)=\left|\rho_{X Y, 12 \cdots n}^{q}-\rho_{X Y}^{q}\right|
$$

We calculate the influence degree function $I(n, q)$ of the synthetical signals using the proposed first-order MFDPCC coefficient and present the influence degree function $I(1, q)$ vs. parameter $q$ in Figure 1. The results of the influence degree values of different $q$ are just about nil, which indicates that there is hardly any effect of white noise sequence on cross-correlation of the two series $X$ and $Y$.

3.2. Stock Market. To further exemplify the potential utility of the $n$-controlling-variables MFDPCC coefficient method for analyzing real-world data, we study daily closing prices of fifteen stock markets including the São Paulo Index (IBOV), the Dow Jones Index (DJI), the NASDAQ Index (IXIC), the Standard \& Poor 500 Composite Stock Price Index (SPX), 


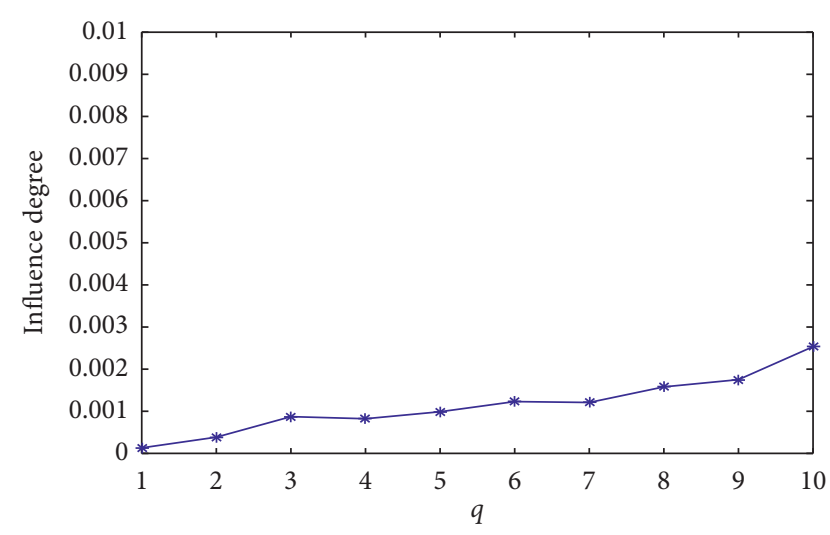

FIGURE 1: The effect of white noise sequence $\xi_{1}$ on cross-correlation of the two series $X$ and $Y$.

the FTSE Global Equity Index Series (FISE), the French CAC 40 (FCHI), German DAX Index (GDAXI), Nikkei 255 Index (N255), Korea Composite Index (KS11), Hang Seng Index (HSI), Australian Standard \& Poor's 200 (AS51), Mumbai Index (SENSEX), Russian Index (RTS), Shanghai Composite Index (SSEC), and Shenzhen Composite Index (SZI). Datasets are from January 04, 1993, to January 03, 2019.

Figure 2 shows the mean of DCCA coefficients for the stock series. The mean of DCCA coefficient between DJI and SPX is 0.97 , which performs relevantly different from other DCCA coefficients. It indicates the close cross-correlation between the American stock markets. The next largest DCCA coefficient $\rho=0.92$ is obtained by SSEC and SZI, which indicates the close cross-correlation in Chinese mainland stock markets.

The mean of DCCA coefficients between SZI and stock markets in developed countries (GDAXI, N225, KS11, and AS51) is less than 0.3. It shows that SZI has a weak relationship with stock markets in developed countries. The mean of DCCA coefficients between SZI and HSI is in an intermediate state, which indicates the existence of crosscorrelation in Chinese stock markets.

Next, we analyze the effect of the other thirteen stock markets on cross-correlation characteristics between SSEC and SZI, by applying the influence degree of first-order DPCC coefficient. For the effect on cross-correlation characteristics between the SSEC and SZI, the largest influence degree $I=0.05$ is obtained by HSI, which shows the information exchange between the Chinese stock market, as seen in Figure 3. The next largest $I=0.04$ is acquired by SENSEX, which indicates the association between the stock markets in developing countries (Indian and Chinese stock markets). The $I$ values of other stock time series are less than 0.1 , which indicates little information exchange between the Chinese mainland stock market and other stock markets. The influence degree values of 13 stock markets for firstorder MFDPCC coefficient with $q=1,2, \ldots, 10$ are also demonstrated in the upper left of Figure 3.

During the analysis, we observe the effect of HSI on cross-correlation characteristics between SSEC and SZI from influence degree function $I(1, q)$ that decreases as the scale $q$ increases. And this infers the change of multifractal crosscorrelation.

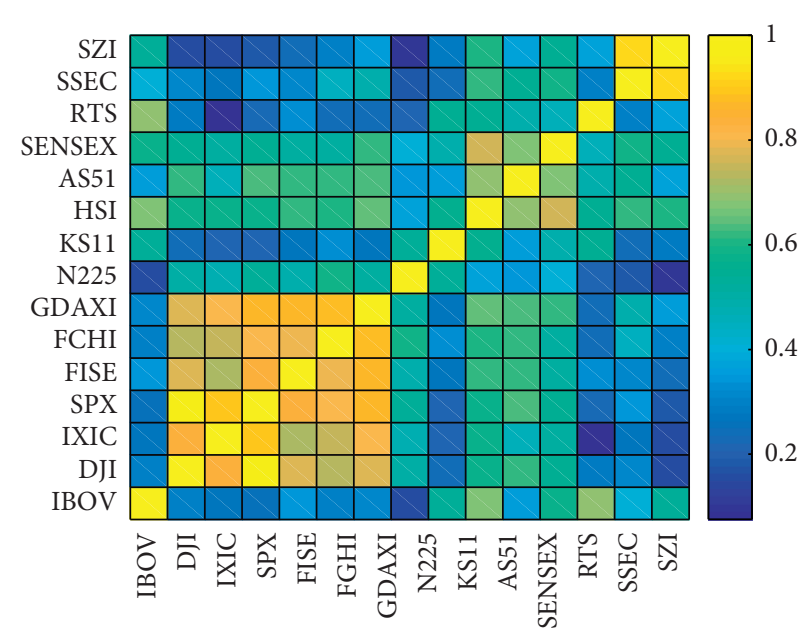

FIgURE 2: The mean of DCCA coefficients between the stock series.

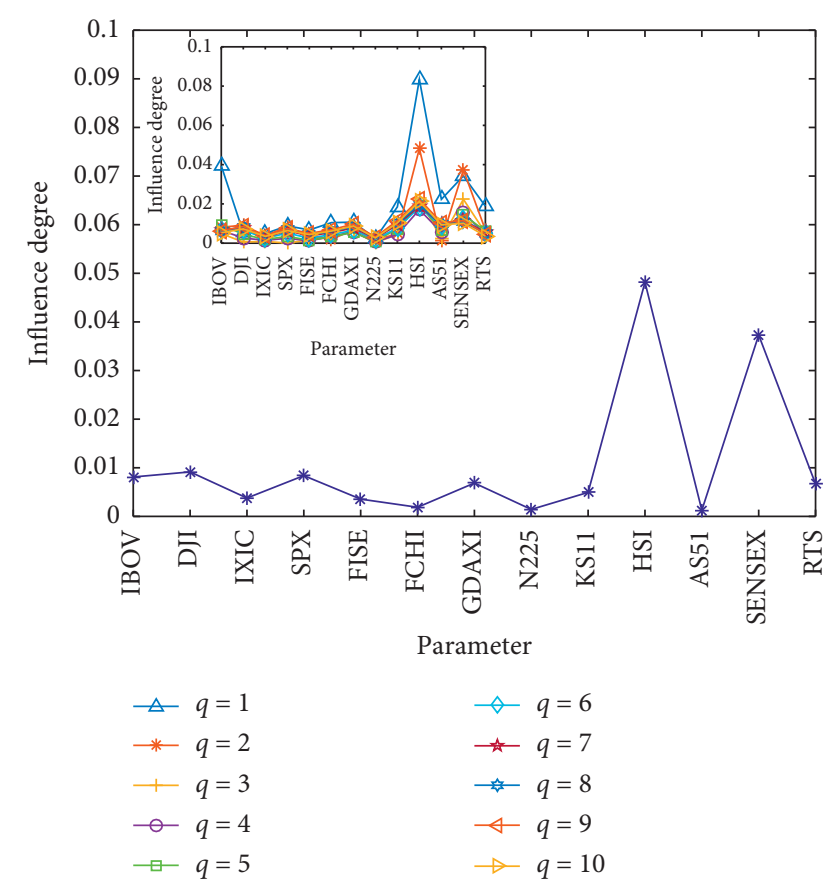

FIgURE 3: The influence degree of the first-order detrended partial cross-correlation in stock market and the influence degree of the first-order multifractal detrended partial cross-correlation coefficient in stock market (inset).

In order to capture the change of multifractal crosscorrelation between two nonstationary time series influenced by common external forces, multifractal detrended partial cross-correlation analysis (MFDPXA) is employed [38]. We also investigate the multifractal behavior between the bivariate time series through MFDPXA method for comparison. The result shows that both the corresponding spectra $f_{x y}(\alpha)$ and $f_{x y, z}(\alpha)$ are wide, but the latter is narrower than the former, which is presented in Figure 4.

Here, we perform cross-correlation analysis using MFDPXA method and give the multifractal spectrum for SZI and SSEC time series in which HSI shows significant 

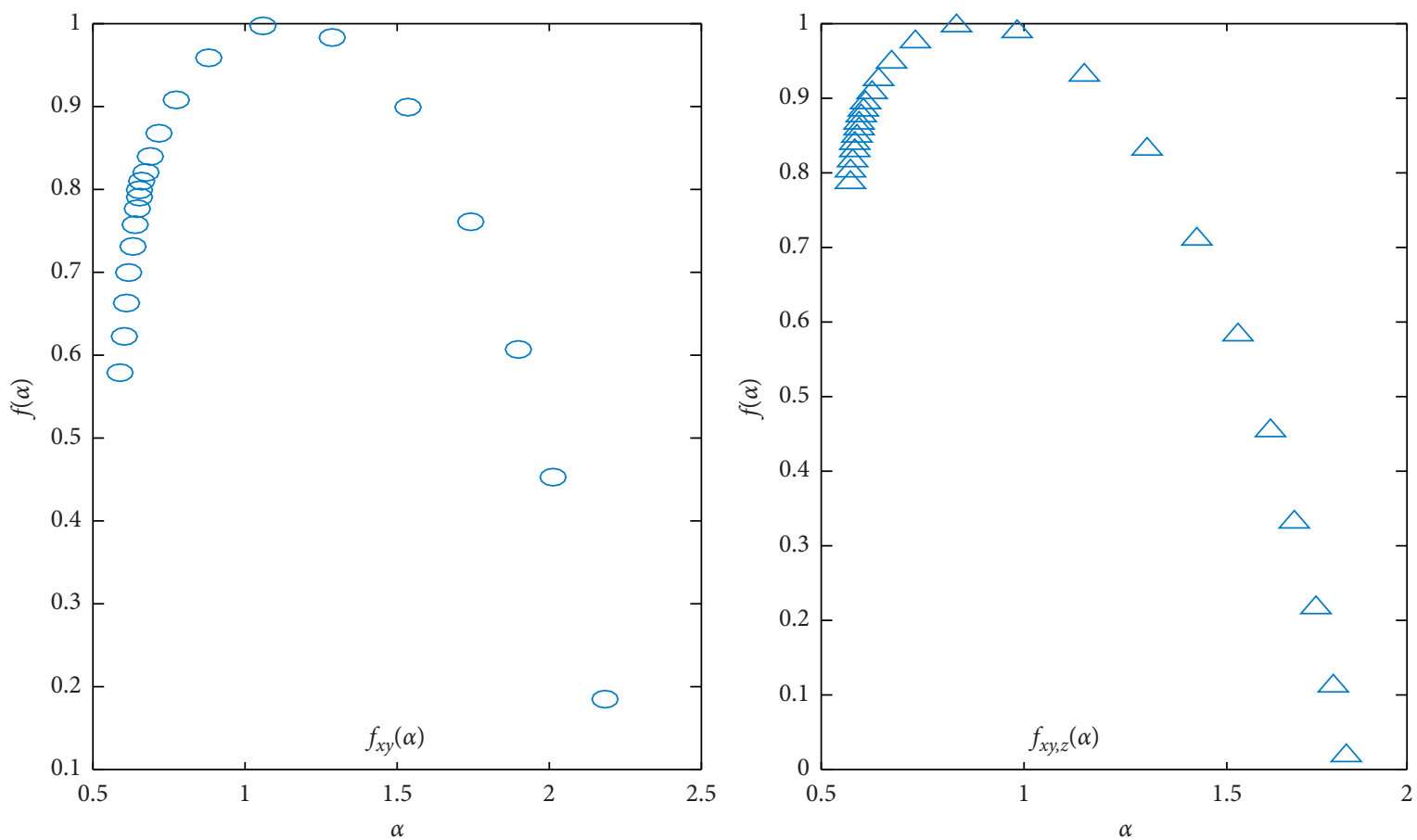

FIGURE 4: The multifractal spectra $f_{x y}(\alpha)$ of bivariate time series obtained through MFDXA method and $f_{x y, z}(\alpha)$ of bivariate time series obtained through MFDPXA, where $x, y$, and $z$ denote the SSEC, SZI, and HSI.

influence on multifractal spectrum, as seen in Figure 4. We compare the obtained influence degrees with the aforementioned method and infer that the HSI has significant influence on SZI and SSEC time series. These similar results imply that the partial cross-correlation method is quite efficient in eliminating external common influence factor.

Applied to scalar variables, the first-order MFDPCC will detect the intrinsic interactions by removing the correlations of controlling variables. When variables are time series, this application is equivalent to removal of zero delay correlations, whereas delayed correlations are not considered $[36,37,40,41]$. Therefore, we investigate the delayed effect of variable $\xi_{1}$ on the correlation between variables $X$ and $Y$. Because the two variables $X$ and $Y$ in question may themselves be correlated at nonzero delays, we write the multifractal detrended partial cross-correlation between $X$ and $Y$, given $\xi_{1}$, as a function of two time delays:

$$
\rho_{X Y\left(\tau_{1}\right), 1\left(\tau_{2}\right)}^{q}=\frac{\rho_{X Y\left(\tau_{1}\right)}^{q}-\rho_{X 1\left(\tau_{2}\right)}^{q} \rho_{Y\left(\tau_{1}\right) 1\left(\tau_{2}\right)}^{q}}{\sqrt{\left(1-\left(\rho_{X 1\left(\tau_{2}\right)}^{q}\right)^{2}\right)\left(1-\left(\rho_{Y\left(\tau_{1}\right) 1\left(\tau_{2}\right)}^{q}\right)^{2}\right)}},
$$

where $\tau_{1}$ is the delay between variables $X$ and $Y$ and $\tau_{2}$ is the delay between variables $X$ and $\xi_{1}$.

In this section, we estimate the delayed effect of HSI on the correlation between SSEC and SZI by using the time delay influence degree $I\left(q, \tau_{1}, \tau_{2}\right)=\left|\rho_{X Y\left(\tau_{1}\right), 1\left(\tau_{2}\right)}^{q}-\rho_{X Y}^{q}\right|$. Figure 5 shows the time delay influence degree for $q=2$. The effect of $\tau_{1}$ on influence degree is weaker than that of $\tau_{2}$ on influence degree.
We now analyze the 2-controlling-variables effect of the other thirteen stock markets on cross-correlation characteristics between SSEC and SZI, by giving a set of two controlling variables. In Figure 6, we illustrate the comparative relation of the influence degree of 2-controllingvariables DPCC coefficients for $13 \times 13$ elements by the matrix diagram.

We note that the structure of the matrix is symmetrical and that element at the intersection of row $i$ and column $j$ represents the influence of controlling variables $\xi_{i}, \xi_{j}$ on the cross-correlation of SSEC and SZI, where the 2-controllingvariables $\xi_{i}, \xi_{j}(i, j=1,2, \ldots, 13)$ are the stock time series from IBOV, DJI, IXIC, SPX, FISE, FCHI, GDAXI, N255, KS11, HSI, AS51, SENSEX, and RTS. Therefore, we analyze the top left corner of the matrix. It can be seen that the largest element is the intersection of row 2 and column 10, i.e., SENSEX and HSI, which indicates the association between the Indian and Chinese stock markets. This is consistent with our result of first-order MFDPCC coefficient method.

Concerning the influence degree $I(2, q)$ of the 2-controlling-variables MFDPCC, we demonstrate 5 cases (HSI and SENSEX, HSI and RTS, SENSEX and KS11, FCHI and N255, and IXIC and FISE) for $q=1,2, \ldots, 10$ in Figure 7. The largest influence degree is the case SENSEX and HSI, which is consistent with the 2-controlling-variables DPCC method.

3.3. Aeroengine Time Series. Previous research studies show that the aeroengine gas path parameters such as lowpressure rotor speed (N1), high-pressure rotor speed (N2), 


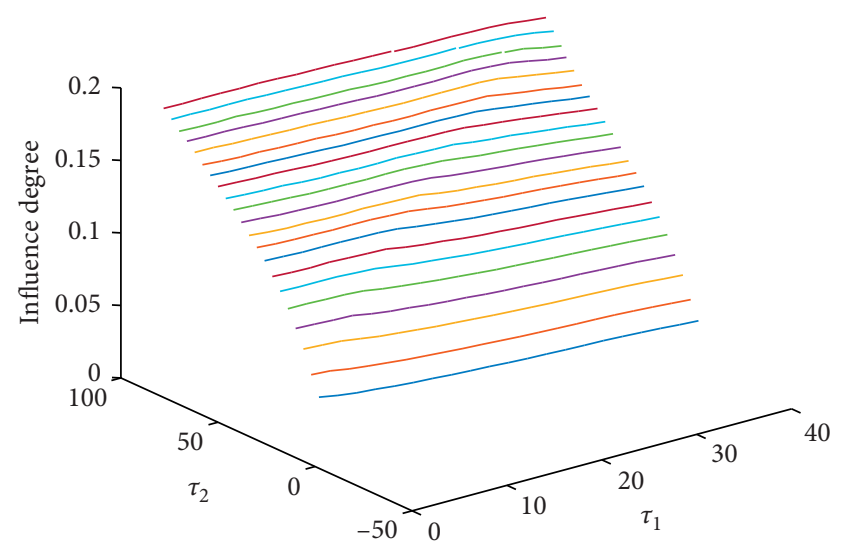

FIGURE 5: The time delay influence degree for delay $\tau_{1}$ between SSEC and SZI and delay $\tau_{2}$ between SSEC and HSI.

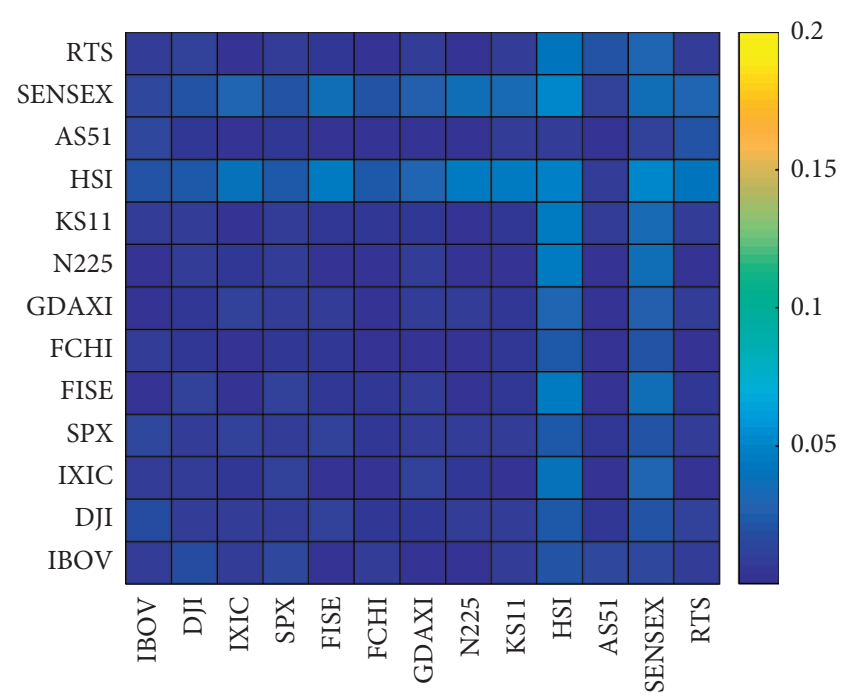

FIGURE 6: The influence degree of the 2-controlling-variables detrended partial cross-correlation.

and fuel flow (WF) play an important role in understanding the aeroengine system [21, 42]. The mean of DCCA coefficients for the aeroengine time series is shown in Figure 8 , where the average DCCA coefficient between $N 1$ and $N 2$ is 0.85 , which shows the close cross-correlation between $N 1$ and $N 2$.

We here investigate the partial correlation between $N 1$ and $\mathrm{N} 2$ given a set of eight controlling variables, including WF, exhaust gas temperature (EGT), N2 tracked vibration channel B (N2TB), inlet air pressure (P2), outlet temperature of high-pressure compressor (T3), outlet temperature of low-pressure compressor (T2.5), and other temperatures (T2 and T2.95).

In Figure 9, we plot the influence degree of first-order DPCC coefficient, investigating the effect of the other eight controlling variables on cross-correlation characteristics between $N 1$ and $N 2$. The largest influence degree $I=0.51$, obtained by T3, shows the information exchange between the outlet temperature of high-pressure compressor and the rotor speed system. The next largest $I=0.22$ is acquired by

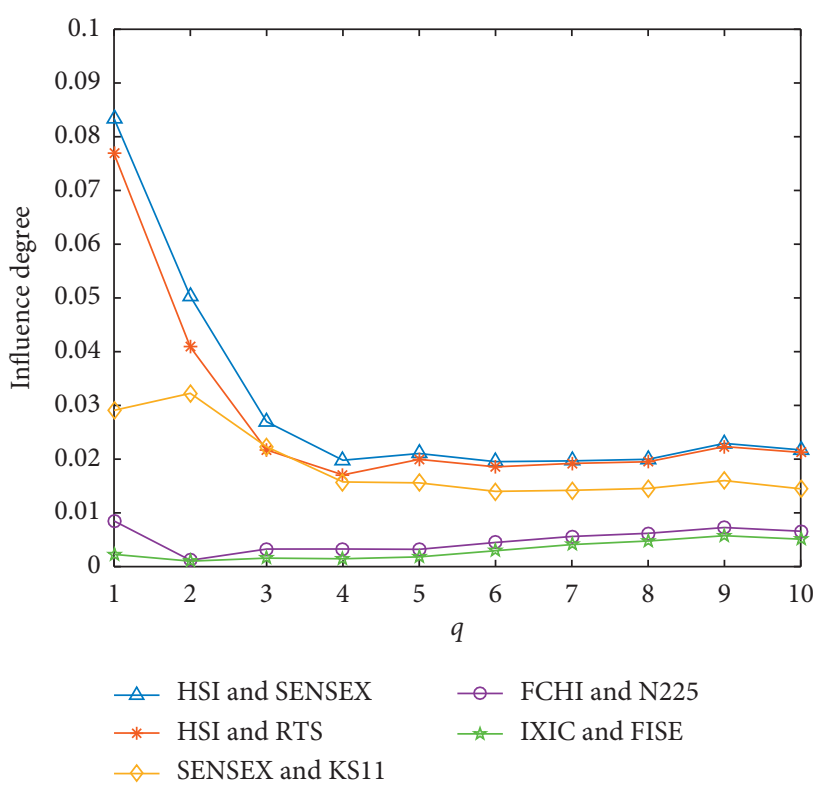

Figure 7: The influence degree of the 2-controlling-variables multifractal detrended partial cross-correlation.

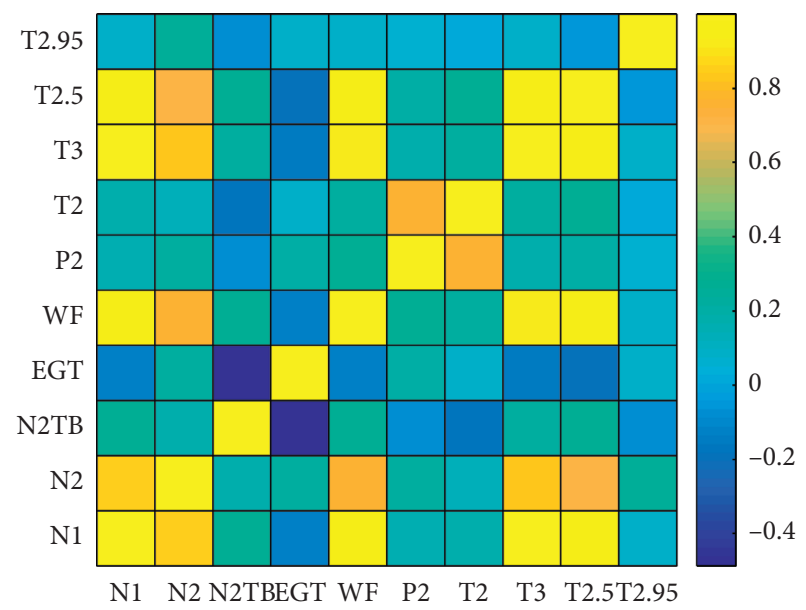

FIGURE 8: The mean of standard DCCA coefficients for the time series of aeroengine system gas path parameters.

WF, which indicates the association between the fuel flow system and rotor speed system.

The result of the influence degree $I(1, q)$ for eight aeroengine parameters applying by first-order MFDPCC coefficient with $q=1,2, \ldots, 10$ is also demonstrated in the upper left of Figure 9. The effect of T3 on cross-correlation characteristics, observed from influence degree function $I(1, q)$, decreases as the scale $q$ increases. It indicates that the multifractal cross-correlation differs across values of $q$.

Further, we apply the MFDPXA method on aforementioned $N 1$ and $N 2$ time series considering the T3 as common influencing factor. It is observed from Figure 10 that the corresponding spectra $f_{x y}(\alpha)$ and $f_{x y, z}(\alpha)$ are wide which shows the strength of multifractal behavior in analyzed time series. We observe that the width of singularity spectrum $f_{x y, z}(\alpha)$ is narrower, and this implies the strength 


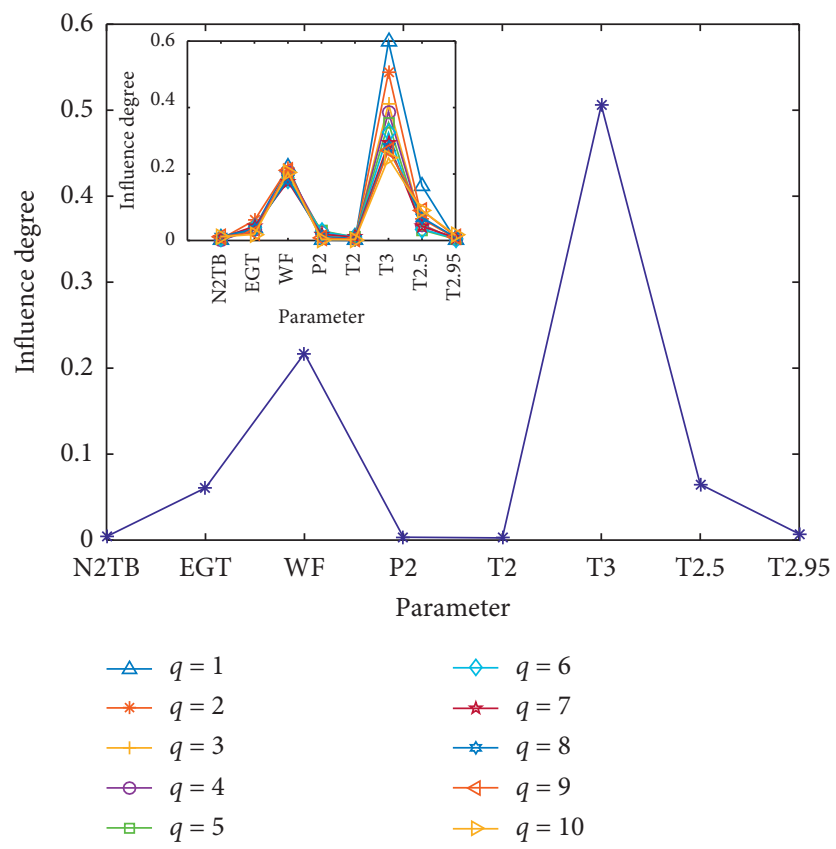

Figure 9: The influence degree of the first-order detrended partial cross-correlation in aeroengine system and the influence degree of the first-order multifractal detrended partial cross-correlation coefficient in aeroengine system (the upper left figure).
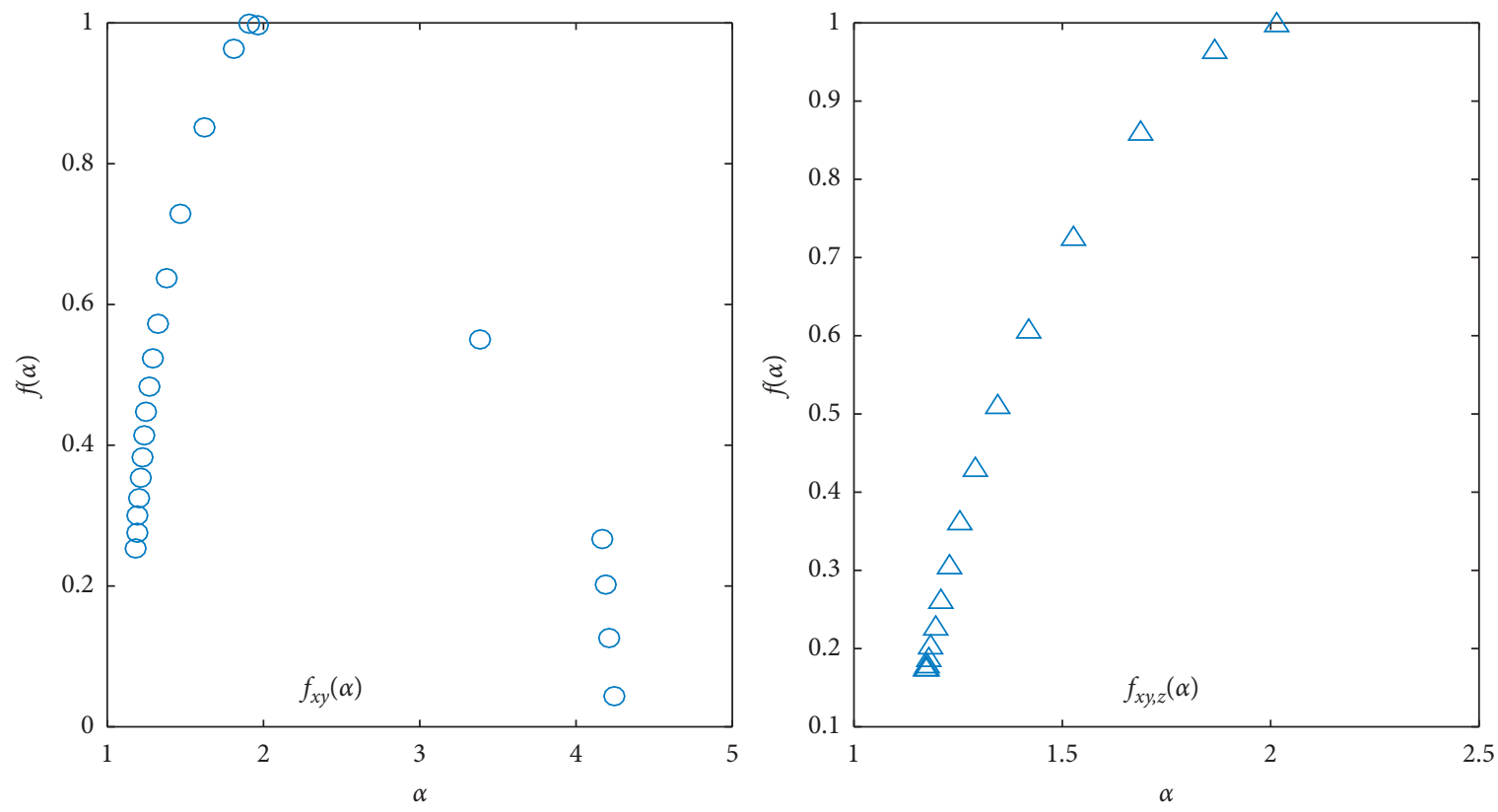

FigURE 10: The multifractal spectrum $f_{x y}(\alpha)$ of bivariate time series obtained through MFDXA method and $f_{x y, z}(\alpha)$ of bivariate time series obtained through MFDPXA, where $x, y$, and $z$ denote $N 1, N 2$, and T3.

of multifractal nature is weak in analyzed bivariate time series.

Here, we estimate the delayed effect of T3 on the correlation between $\mathrm{N} 1$ and $\mathrm{N} 2$ by using the time delay influence degree $I\left(q, \tau_{1}, \tau_{2}\right)$. Figure 11 shows the time delay influence degree for $q=2$. It is obvious that the time delay influence degree gradually increases and then declines as a single-peak curve when $\tau_{2}$ remains constant. As $\tau_{2}$ increases, the peak value of time delay influence degree shifts rightward.

The next observation concerns the influence degree of 2-controlling-variables DPCC coefficient in the aeroengine system. We now analyze the influence of two controlling parameters on the cross-correlation between $N 1$ and $N 2$. In Figure 12, we illustrate the comparative relation of the influence degree of 2-controlling-variables DPCC 


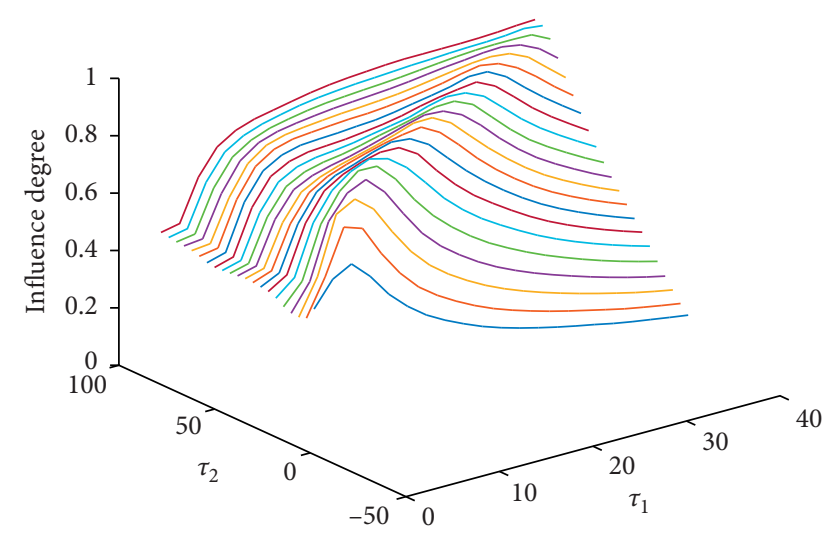

FIgURE 11: The time delay influence degree for delay $\tau_{1}$ between $N 1$ and $N 2$ and delay $\tau_{2}$ between $N 1$ and T3.

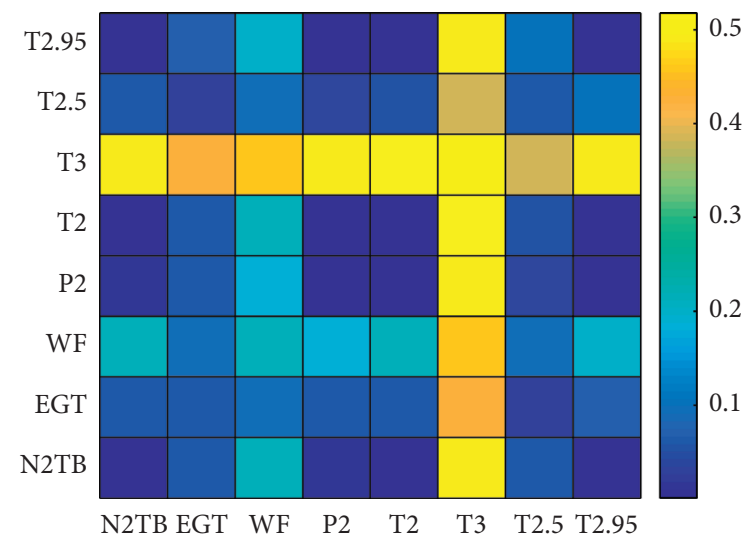

FIGURE 12: The influence degree of the 2-controlling-variables detrended partial cross-correlation for aeroengine system.

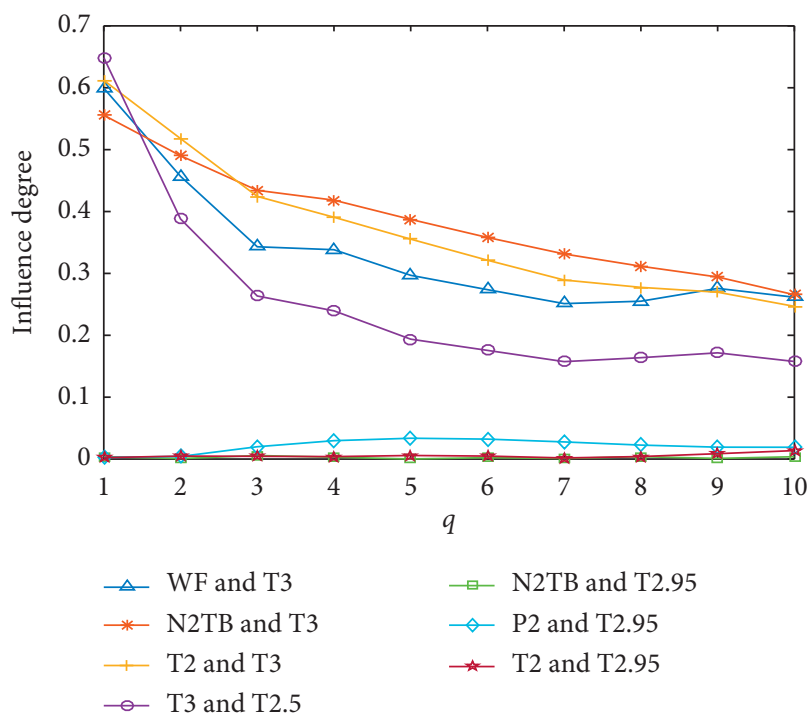

Figure 13: The influence degree of the 2-controlling-variables multifractal detrended partial cross-correlation for the aeroengine system.

coefficient for aeroengine system. It can be seen that the larger elements in the symmetrical matrix are located at row 3 or column 6 , which denote T3 has a greater impact on the correlation between $N 1$ and $N 2$.
Concerning the influence degree $I(2, q)$ of the 2-controlling-variables MFDPCC, we demonstrate 7 cases (T3 and WF, T3 and N2TB, T3 and T2, T3 and T2.5, T2.95 and N2TB, T2.95 and P2, and T2.95 and T2) for $q=1,2, \ldots, 10$ in Figure 13. Larger influence degrees exist in the cases with the presence of T3 (T3 and WF, T3 and N2TB, T3 and T2, and T3 and T2.5), which is consistent with the 2-controllingvariables DPCC method, as seen in Figure 12.

For the aeroengine, the parameters $N 1$ and $N 2$ are chosen to indicate the engine thrust which depends on the throttle lever angle. Hence, the cross-correlation between them is strong. The temperature and pressure parameters are linked with many factors, including the compressor power, combustion efficiency, throttle lever angle, etc. Therefore, the dynamic interaction of these three groups makes the aeroengine function. These results estimate the influence of temperature and pressure parameters on the cross-correlation between $N 1$ and $N 2$.

\section{Conclusion}

In this paper, we propose the $n$ th-order multifractal detrended partial cross-correlation analysis method and the $n$-controlling-variables multifractal detrended partial crosscorrelation analysis method for understanding the interactions between two nonstationary time series. For comparing these new methods with classical measures, we introduce the influence degree function. We then apply the $n$-controlling-variables multifractal detrended partial crosscorrelation analysis of stock markets and aeroengine performance parameters and measure the influence degree function of the partial cross-correlation in a dynamic system.

To understand the numerous real-world systems where the output signals exhibit complex cross-correlation, both cross-correlation and partial correlation are subjects of investigation. The information of $n$-variables MFDPCC helps people to research information exchange in complex systems. This paper gives two examples, stock markets and aeroengine systems. For stock time series, our results indicate that, concerning closing index values, there is little information exchange between the Chinese stock markets and the American-European stock markets, whereas the SSEC, SZI, and HSI, by first-order MFDPCC method and 2controlling-variables MFDPCC, show frequent and abundant information exchange in Chinese stock markets. For aeroengine performance parameters, our results show that there is some information exchange between the engine rotor system and the aeroengine parameters, such as the outlet temperature of the high-pressure compressor and the fuel flow.

We believe that the MFDPCC method can be used to detect the intrinsic interactions among multiple dynamical systems, and therefore it can be widely applied to many research fields such as the aeroengine health monitoring systems and the investment portfolio where the covariance is employed to explore the interaction of assets income.

The multifractal detrended partial cross-correlation analysis is used to delete the possible indirect correlation, but it may also delete valuable information. This problem 
required further investigation, both experimental and theoretical. Hence, the results of this paper should be considered as preliminary results on the multifractal detrended partial cross-correlation analysis. Therefore, we hope that this study will be extended to analyze the filtered information.

\section{Data Availability}

The stock market data used to support the findings of this study are available from the corresponding author upon request. The aeroengine data used to support the findings of this study have not been made available because of commercial secrets.

\section{Conflicts of Interest}

The authors declare that they have no conflicts of interest.

\section{Acknowledgments}

The financial support from the funds of the MOE (Ministry of Education in China) Project of Humanities and Social Sciences under grant no. 19YJC910001 and the Fundamental Research Funds for the Central Universities under grant no. 3122014 K013 is gratefully acknowledged.

\section{References}

[1] K. Pearson, "Note on regression and inheritance in the case of two parents," Proceedings of the Royal Society, vol. 58, pp. 240-242, 1895.

[2] K. Pearson, "On lines and planes of closest fit to systems of points in space," The London, Edinburgh, and Dublin Philosophical Magazine and Journal of Science, vol. 2, no. 11, pp. 559-572, 1901.

[3] E. P. Wigner, "Characteristic vectors of bordered matrices with infinite dimensions," The Annals of Mathematics, vol. 62, no. 3, pp. 548-564, 1955.

[4] G. Golub and W. Kahan, "Calculating the singular values and pseudo-inverse of a matrix," Journal of the Society for Industrial and Applied Mathematics Series B Numerical Analysis, vol. 2, no. 2, pp. 205-224, 1965.

[5] B. Podobnik and H. E. Stanley, "Detrended cross-correlation analysis: a new method for analyzing two nonstationary time series," Physical Review Letters, vol. 100, Article ID 084102, 2008.

[6] G. F. Zebende, "DCCA cross-correlation coefficient: quantifying level of cross-correlation," Physica A: Statistical Mechanics and Its Applications, vol. 390, no. 4, pp. 614-618, 2011.

[7] A. Machado Filho, M. F. da Silva, and G. F. Zebende, "Autocorrelation and cross-correlation in time series of homicide and attempted homicide," Physica A: Statistical Mechanics and Its Applications, vol. 400, pp. 12-19, 2014.

[8] C. Xue, P. Shang, and W. Jing, "Multifractal detrended crosscorrelation analysis of BVP model time series," Nonlinear Dynamics, vol. 69, no. 1-2, pp. 263-273, 2012.

[9] R. T. Vassoler and G. F. Zebende, "DCCA cross-correlation coefficient apply in time series of air temperature and air relative humidity," Physica A: Statistical Mechanics and Its Applications, vol. 391, no. 7, pp. 2438-2443, 2012.
[10] S. Shadkhoo and G. R. Jafari, "Multifractal detrended crosscorrelation analysis of temporal and spatial seismic data," The European Physical Journal B, vol. 72, no. 4, pp. 679-683, 2009.

[11] E. B. S. Marinho, A. M. Y. R. Sousa, and R. F. S. Andrade, "Using detrended cross-correlation analysis in geophysical data," Physica A: Statistical Mechanics and Its Applications, vol. 392, no. 9, pp. 2195-2201, 2013.

[12] K. Dong, P. Shang, and A. Lin, "Chaotic SVD method for minimizing the effect of seasonal trends in detrended crosscorrelation analysis," DCDIS Series B: Applications \& Algorithms, vol. 18, pp. 261-277, 2011.

[13] X. Zhao, P. Shang, A. Lin, and G. Chen, "Multifractal Fourier detrended cross-correlation analysis of traffic signals," Physica A: Statistical Mechanics and Its Applications, vol. 390, no. 2122, pp. 3670-3678, 2011.

[14] R. A. Ribeiro, M. V. M. Mata, L. S. Lucena, U. L. Fulco, and G. Corso, "Spatial analysis of oil reservoirs using detrended fluctuation analysis of geophysical data," Nonlinear Processes in Geophysics, vol. 21, no. 5, pp. 1043-1049, 2014.

[15] Q. Fan and D. Li, "Multifractal cross-correlation analysis in electricity spot market," Physica A: Statistical Mechanics and Its Applications, vol. 429, pp. 17-27, 2015.

[16] X. Zhao, P. Shang, and W. Shi, "Multifractal cross-correlation spectra analysis on Chinese stock markets," Physica A: Statistical Mechanics and Its Applications, vol. 402, pp. 84-92, 2014.

[17] B. Podobnik, D. Horvatic, A. M. Petersen, and H. E. Stanley, "Cross-correlations between volume change and price change," Proceedings of the National Academy of Sciences, vol. 106, no. 52, pp. 22079-22084, 2009.

[18] B. Podobnik, Z. Q. Jiang, W. X. Zhou, and H. E. Stanley, "Statistical tests for power-law cross-correlated processes," Physical Review E, vol. 84, p. 66118, 2011.

[19] I. Gvozdanovic, B. Podobnik, D. Wang, and H. Eugene Stanley, "1/f behavior in cross-correlations between absolute returns in a US market," Physica A, vol. 391, pp. 2860-2866, 2012.

[20] X. Y. Qian, Y. M. Liu, Z. Q. Jiang, B. Podobnik, W. X. Zhou, and H. E. Stanley, "Detrended partial cross-correlation analysis of two time series influenced by common external forces," Physical Review E, vol. 91, Article ID 062816, 2015.

[21] K. Dong, Y. Gao, and C. Zhu, "Aero engine data correlation by means of detrended fluctuation analysis," International Review of Aerospace Engineering, vol. 5, pp. 251-255, 2012.

[22] K. Dong, J. Fan, and Y. Gao, "Cross-correlations and structures of aero-engine gas path system based on dcca coefficient and rooted tree," Fluctuation and Noise Letters, vol. 14, p. 1550014, 2015.

[23] K. Dong, Y. Gao, and L. Jing, "Correlation tests of the engine performance parameter by using the detrended cross-correlation coefficient," Journal of the Korean Physical Society, vol. 66, pp. 539-543, 2015.

[24] K. Dong, Y. Gao, and N. Wang, "EMD method for minimizing the effect of seasonal trends in detrended cross-correlation analysis," Mathematical Problems in Engineering, vol. 2013, Article ID 493893, 7 pages, 2013.

[25] P. Baranowski, J. Krzyszczak, C. Slawinski et al., "Multifractal analysis of meteorological time series to assess climate impacts," Climate Research, vol. 65, pp. 39-52, 2015.

[26] N. Kalamaras, K. Philippopoulos, D. Deligiorgi, C. G. Tzanis, and G. Karvounis, "Multifractal scaling properties of daily air temperature time series," Chaos, Solitons and Fractals, vol. 98, pp. 38-43, 2017. 
[27] J. Ei, J. Zhang, X. Liu, and F. Li, "Multi-fractal scaling comparison of the air temperature and the surface temperature over China," Physica A, vol. 462, pp. 783-792, 2016.

[28] S. Debdeep, R. Rinku, and M. Manjunatha, "Epilepsy and seizure characterisation by multifractal analysis of EEG subbands," Biomedical Signal Processing, vol. 41, pp. 264-270, 2018.

[29] S. Bayraci, "Testing for multi-fractality and efficiency in selected sovereign bond markets: a multi-fractal detrended moving average (MF-DMA) analysis," International Journal of Computational Economics and Econometrics, vol. 8, pp. 95-120, 2018.

[30] Z. Jiang, W. Xie, W. Zhou, and D. Sornette, "Multifractal analysis of financial markets: a review," Reports on Progress in Physics, vol. 82, no. 12, p. 125901, 2019.

[31] C. Yao, C. Liu, and W. Ju, "Multifractal analysis of the WTI crude oil market, US stock market and EPU," Physica A, vol. 550, p. 124096, 2020.

[32] W. Zhou, "Multifractal detrended cross-correlation analysis for two nonstationary signals," Physical Review E, vol. 77, Article ID 066211, 2008.

[33] C. Tzanis, I. Koutsogiannis, K. Philippopoulos, and N. Kalamaras, "Multifractal detrended cross-correlation analysis of global methane and temperature," Remote Sensing, vol. 12, p. 557, 2020.

[34] C. Zhang, Z. Ni, and L. Ni, "Multifractal detrended crosscorrelation analysis between PM2.5 and meteorological factors," Physica A, vol. 438, pp. 114-123, 2015.

[35] K. Pearson, "On some novel properties of partial and multiple correlation coefficients in a universe of manifold characteristics," Biometrika, vol. 11, pp. 231-1238, 1916.

[36] S. Eran, D. Rotem, and A. Moshe, "Partial cross-correlation analysis resolves ambiguity in the encoding of multiple movement features," Journal of Neurophysiology, vol. 95, pp. 1966-1975, 2006.

[37] N. Zhang, A. Lin, and P. Yang, "Detrended moving average partial cross-correlation analysis on financial time series," Physica A, vol. 542, p. 122960, 2020.

[38] H. Sri Sai, M. Pal, and P. Manimaran, "Multifractal detrended partial cross-correlation analysis on Asian markets," Physica A, vol. 531, Article ID 121778, 2019.

[39] B. Podobnik, D. Horvatic, A. L. Ng, H. E. Stanley, and P. C. Ivanov, "Modeling long-range cross-correlations in twocomponent ARFIMA and FIARCH processes," Physica A, vol. 387, pp. 3954-3959, 2008.

[40] K. Dong, L. Long, H. Zhang, and X. Su, "The Lempel-Ziv measure based pedigree map to detect and evaluate correlation between aero-engine gas path system variables," Physica A, vol. 525, pp. 1080-1087, 2019.

[41] Y. Song, C. Kube, J. Zhang, and X. Li, "Higher-order spatial correlation coefficients of ultrasonic backscattering signals using partial cross-correlation analysis," The Journal of the Acoustical Society of America, vol. 147, pp. 757-768, 2020.

[42] K. Dong, H. Zhang, and Y. Gao, "Dynamical mechanism in aero-engine gas path system using minimum spanning tree and detrended cross-correlation analysis," Physica A, vol. 465, pp. 363-369, 2017. 